\title{
Reduction of Convective Losses in Solar Cavity Receivers
}

\author{
Graham Hughes ${ }^{1, \text { a) }}$, John Pye ${ }^{2, \text { b) }}$, Martin Kaufer ${ }^{2, c)}$, Ehsan Abbasi-Shavazi ${ }^{2, \text { d), }}$ \\ Jack Zhang ${ }^{2}$, Adam McIntosh ${ }^{2}$ and Tim Lindley ${ }^{2}$
}

\author{
${ }^{1}$ Research School of Earth Sciences, Australian National University, Canberra, ACT 2601 Australia \\ ${ }^{2}$ Solar Thermal Group, College of Engineering and Computer Science, Australian National University, Canberra, \\ ACT 2601 Australia
}

${ }^{a)}$ Corresponding author: graham.hughes@anu.edu.au

b)john.pye@anu.edu.au

c) martin.kaufer@anu.edu.au

d) ehsan.abbasi@anu.edu.au

\begin{abstract}
Two design innovations are reported that can help improve the thermal performance of a solar cavity receiver. These innovations utilise the natural variation of wall temperature inside the cavity and active management of airflow in the vicinity of the receiver. The results of computational fluid dynamics modelling and laboratory-scale experiments suggest that the convective loss from a receiver can be reduced substantially by either mechanism. A further benefit is that both radiative and overall thermal losses from the cavity may be reduced. Further work to assess the performance of such receiver designs under operational conditions is discussed.
\end{abstract}

\section{INTRODUCTION}

The capture of incident solar energy with a cavity receiver situated near the focus of a much larger collection area is an established technology in high-temperature concentrating solar thermal systems. Examples include the ANU Big Dish [1] and central tower systems [2]. The cavity geometry of the receiver helps to reduce thermal losses to the environment, and to shield those surfaces from external influences such as the ambient wind that can act to lower thermal performance. The cavity geometry can also enhance energy capture in situations where the receiver orientation is not fixed, for instance in a concentrating dish system where the collection surface tracks the sun.

The surface temperature distribution inside the cavity is the result of a local balance between the net radiation, convection and rate at which heat is harnessed - often by conduction through the receiver wall into a heat transfer fluid. Thus the main mechanisms of heat loss from the receiver - thermal emission and reflection from the interior surfaces and transport by air heated inside the cavity - involve passage of thermal energy through the aperture to the external environment. One approach to reduce these losses is to glaze the cavity aperture with an optically transparent material such as a quartz or sapphire window [3,4,5], which both traps radiation re-reradiated at longer (infrared) wavelengths and acts as a physical barrier to the exchange of air with the cavity. However, such a window imposes significant limitations on the physical conditions (i.e. temperature, pressure, cleanliness) under which a cavity receiver can be operated. In this study, we instead concentrate on a cavity receiver that has an unglazed aperture and is therefore partially open to the atmosphere. The material properties of the cavity and heat transfer fluid, and the optics of the external collection area, typically determine the design constraints for the receiver.

Previous work shows that receiver losses due to radiation and convection are coupled and can both be significant [6], and a companion paper [7] considers how cavity geometry can be optimised to minimise the overall loss. However, the goal of the current work is to develop a better physical understanding of the convective component of loss, and to examine whether these losses can be controlled. In order to achieve this aim, we choose to simplify the problem by imposing the temperature field inside a given cavity receiver, thus decoupling the radiation and convection (and geometry optimisation) problems. 
We report two innovations that can be used to improve cavity receiver design. Firstly, we demonstrate how the natural variation of inner cavity wall temperature in a cavity can be used to recoup heat that would otherwise be lost. Secondly, we consider how airflow in the vicinity of the receiver can be actively managed to disrupt the convective flow that would otherwise occur. The results of computational fluid dynamics (CFD) modelling and laboratory-scale experiments suggest that either feature can substantially reduce the overall thermal loss from the cavity. We outline further work to assess the performance of such receiver designs under operational conditions.

\section{INTERNAL RECUPERATION OF CONVECTIVE LOSSES}

We consider the effect on convective flows of a non-uniform temperature distribution over the interior walls of the receiver. Such non-uniformity is dependent upon a number of factors, including the properties and path of the heat transfer fluid through the receiver and the distribution of incident radiation passing through the cavity aperture from the external collection surface. Moreover, local thermal emission losses to the external environment tend to be higher near the cavity aperture. Many previous studies of convection in cavity receivers (e.g. $[8,9,10,11]$ ) have simplified the problem by imposing either a uniform temperature or a heat flux distribution on the inner cavity surface, thus decoupling the radiative and convective heat transfer problems. In this part of the current study, we also maintain a decoupling between the two heat transfer mechanisms, but instead impose a temperature distribution that is non-uniform (as suggested by the simplified, but integrated, modelling of thermal emissions and the heat transfer fluid [6]). The rationale for this approximate decoupling is that the local thermal emission will determine the local wall temperature $T$ to leading order because the emission depends on $T$ raised to the fourth power. As we shall see, the additional degree of freedom permitted here by our use of an imposed non-uniform temperature distribution leads to new physical insights into convective loss.

\section{Computational Fluid Dynamics Modelling}

A set of three-dimensional high-resolution CFD simulations was conducted for the ANU SG3 cavity receiver that has previously been the subject of measurement and modelling effort (e.g. [9,12]). The geometry has a "top-hat" shape (Fig. 1), consisting of an approximately cylindrical section of inner diameter $0.68 \mathrm{~m}$ and length $1.245 \mathrm{~m}$ that is closed at one end and abuts the flared "brim" (outer diameter $1.5 \mathrm{~m}$ ) of the hat at the aperture end. The receiver geometry was embedded in a significantly larger "ambient" domain, which had open boundaries at the top and bottom. The overall computational domain was discretised in an unstructured grid using up to 13.5 million tetrahedral elements, the bulk of which were situated inside or near the aperture of the receiver and with the maximum resolution (as fine as $0.002 \mathrm{~m}$ ) in the boundary layers adjacent to the receiver walls. The highly-resolved computations did not require a turbulence closure scheme. The simulations were carried out by iterating the compressible and transient OpenFOAM solver ("buoyantPimpleFoam") for more than $300 \mathrm{~s}$, by which time the flow had reached thermal equilibrium.

We present results from two simulations here - a control case in which the receiver wall is held at $793 \mathrm{~K}$ everywhere (Fig. 1b), and another in which the imposed wall temperature decreases from a similar maximum $(816 \mathrm{~K})$ at the innermost cavity to $332 \mathrm{~K}$ near the flared aperture (Fig. 1a). The temperature distribution in the latter case is predicted from a model [6] that calculates at every point a leading order balance between net thermal radiation and heat transport to a heat transfer fluid, which enters the receiver at the outer aperture, passes along a small diameter tube coiled to form the receiver wall, and exits at the top of the cylindrical section. No-slip boundary conditions are also imposed on the receiver walls, and the receiver axis is inclined at $40^{\circ}$ to the horizontal in both examples shown here. The ambient temperature was set to $293 \mathrm{~K}$.

The results demonstrate significant differences in heat loss and flow structure associated with the non-uniform temperature boundary conditions. A uniform wall temperature leads to a relatively stagnant pool of hot air trapped in the upper reaches of the cavity, as has been observed in a number of previous studies (e.g. $[8,13])$; this region is apparent in Fig. 1d where the heat flux is small. However, this region does not remain stagnant when the wall temperature is non-uniform. The air is both heated and cooled there, as evidenced by wall heat fluxes of opposite sign in Fig. 1c, and a stronger convective circulation (not shown) is found to exist in and extend beyond this region. In general for a non-uniform temperature distribution, heat loss occurs to air in the receiver over a portion of the interior cavity surface, but a significant fraction of this loss can be recuperated in regions where the surface is cooler than the heated air (Fig. 1c). This recuperation mechanism is absent when the wall temperature is assumed uniform. For instance, the overall convective loss is approximately $1.6 \mathrm{~kW}$ for the case in Fig. 1a, c - this convective loss is 
about $12 \%$ of that for the control case (approximately $13.4 \mathrm{~kW}$; Fig. 1b, d) in which the imposed wall temperature is uniform but with a similar maximum, or is comparable to that computed with a uniform imposed wall temperature of just $393 \mathrm{~K}$ (approximately $2 \mathrm{~kW}$; not shown here).

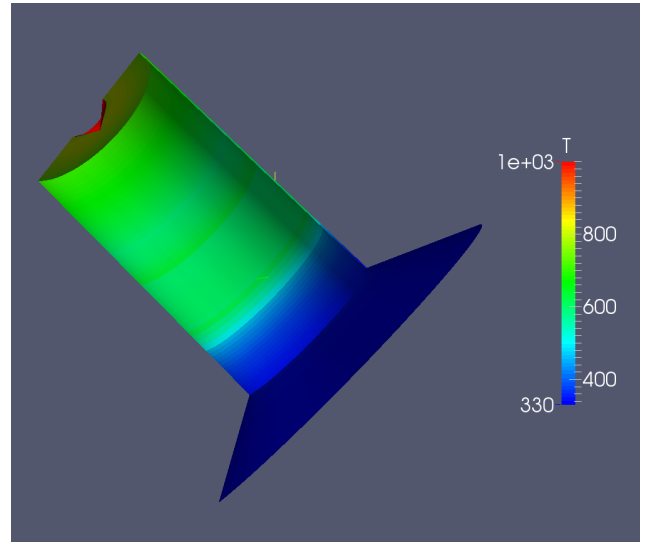

(a)

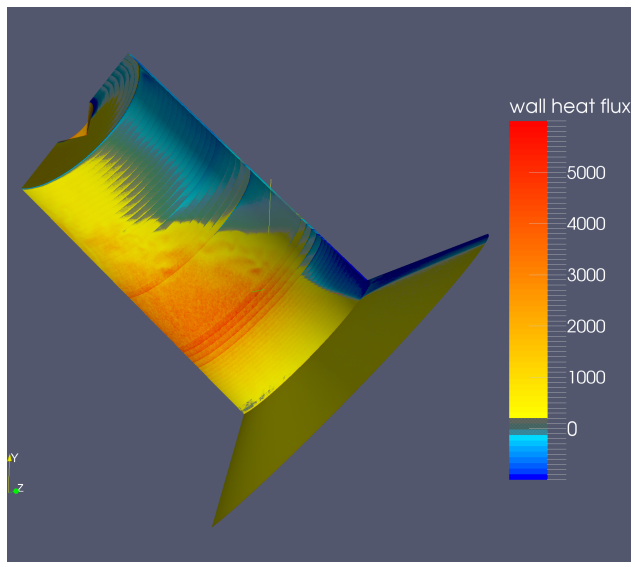

(c)

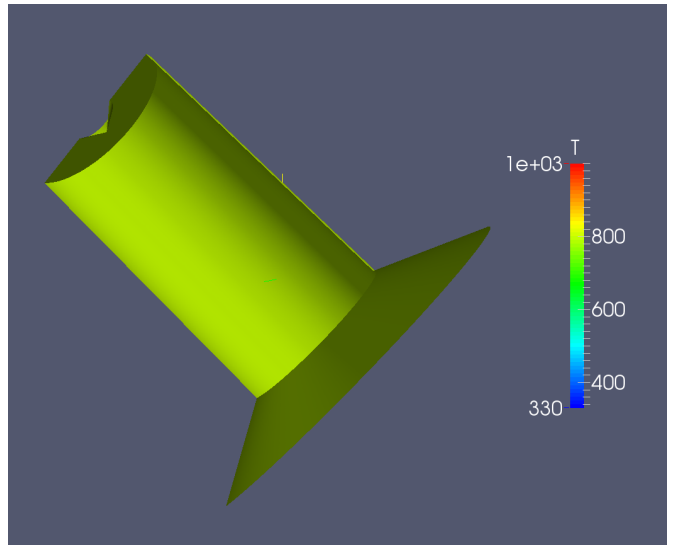

(b)

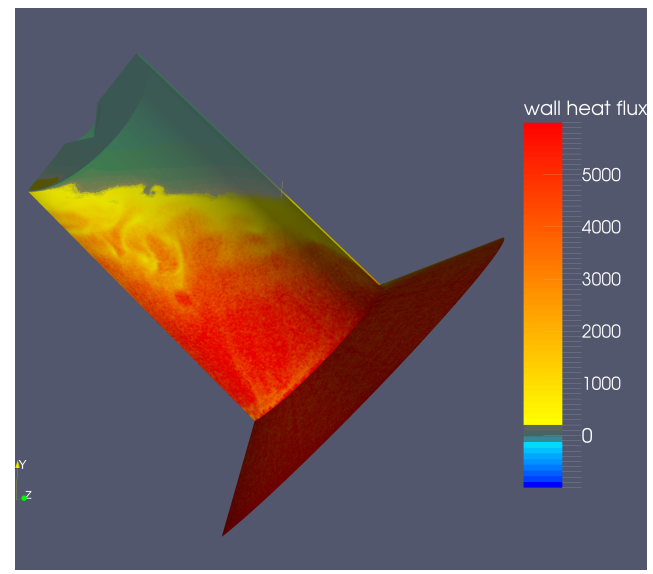

(d)

FIGURE 1. Example results from two CFD simulations of cavity receiver convection (based on the SG3 cavity receiver on the ANU Big Dish) with: (a) imposed non-uniform wall temperature (K) profile, obtained from a radiatively-coupled model (see [6] for details) that incorporates the heat transfer fluid (water is introduced at the aperture end of the receiver at $332 \mathrm{~K}$ and extracted as supercritical steam at enclosed end of the cavity.); (b) imposed uniform wall temperature, such that the maximum wall temperature is similar to (a); (c) the wall heat flux $\left(\mathrm{W} \mathrm{m}^{-2}\right)$ corresponding to (a); (d) the wall heat flux corresponding to (b). The cavity axis is inclined at $40^{\circ}$ from the horizontal and positive heat flux is defined as a transfer from the cavity wall to the air. Note that the minimum radius to which the tube carrying the heat transfer fluid in the SG3 receiver may be coiled gives rise to the small region of elevated temperature (approximately $1050 \mathrm{~K}$ ) in the centre of the upper end of the cavity in both simulations.

\section{Laboratory Experiments}

An experimental program has investigated the convective heat loss from a scale-model cavity receiver under controlled laboratory conditions. The model receiver is a cylindrical stainless steel cavity of diameter $83 \mathrm{~mm}$ with a length of either $83 \mathrm{~mm}$ or $166 \mathrm{~mm}$. The cavity is electrically heated by a resistance cable wound around the outer wall. Thermocouples were embedded in the wall at numerous locations throughout the cavity; a general description of the apparatus construction, instrumentation and methodology may be found in [14]. Two configurations are reported here for which the mean cavity temperature was maintained at approximately $450^{\circ} \mathrm{C}$. Heating is applied at a known rate along the length of the cylindrical cavity whose length and diameter are equal (schematic not shown here - see [14] for details). In the second case, heating is applied at a known rate (which turns out to be similar to the first case) along the innermost half of the cylindrical cavity with length to diameter ratio of two (see Fig. 2a); the "passive" outermost half of the cavity is uninsulated and exposed to the ambient environment. This second 
configuration is used to demonstrate qualitatively the recuperation effect introduced above: some of the heat supplied is transferred through the outermost section of cavity wall rather than being all lost by convection and radiation through the aperture. The inclination angle that the cavity axis makes with the horizontal is varied from $0^{\circ}$ to $90^{\circ}$.

Measurement of the cavity wall temperature field and the heating power input together with models for conductive and radiative losses from the cavity allow the convection losses to be calculated (Fig. 2b; see [14] for complete details). Note that for the purposes of the analysis here, the heat supply rate is reduced by the rate of conductive losses into the cavity housing because they are of no dynamical significance. However, the heat that can be recuperated by virtue of the reduced wall temperatures in the passive section case is calculated by applying a conduction analysis to this region, based on the wall temperature measurements and the known material properties and dimensions. Figure $2 \mathrm{~b}$ shows a substantial reduction in losses associated with both the radiative and convective components; typically more than $50 \%$ of the heat supplied is recuperated. The reduction in losses observed here cannot be explained as simply a result of changing the length to diameter ratio of the cavity from one to two. Preliminary experiments using a further cavity configuration not shown here (geometry as shown in Fig. 2a, but with heating along the entire wall length $L$ ) suggest that the reduction in aperture losses attributable to the different length to diameter ratio is typically of order $10 \%$, and therefore much less than the relative changes measured in Fig. $2 \mathrm{~b}$.

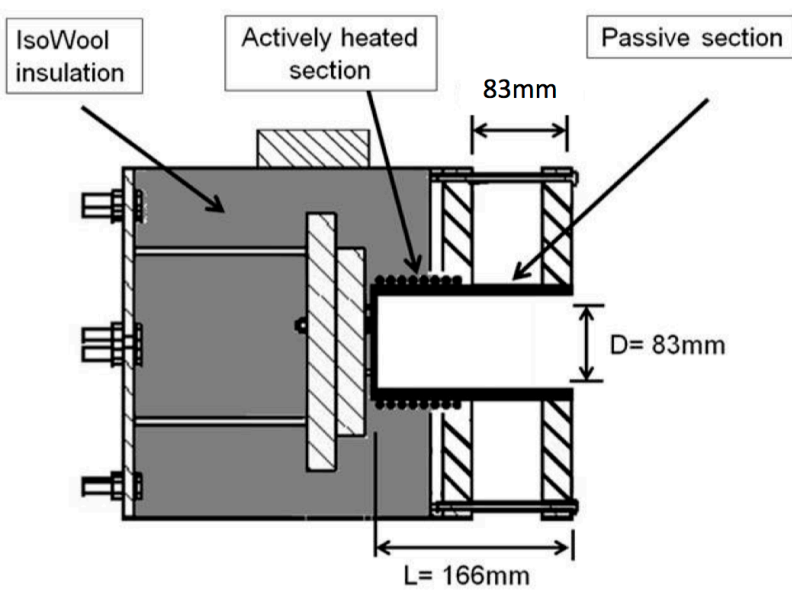

(a)

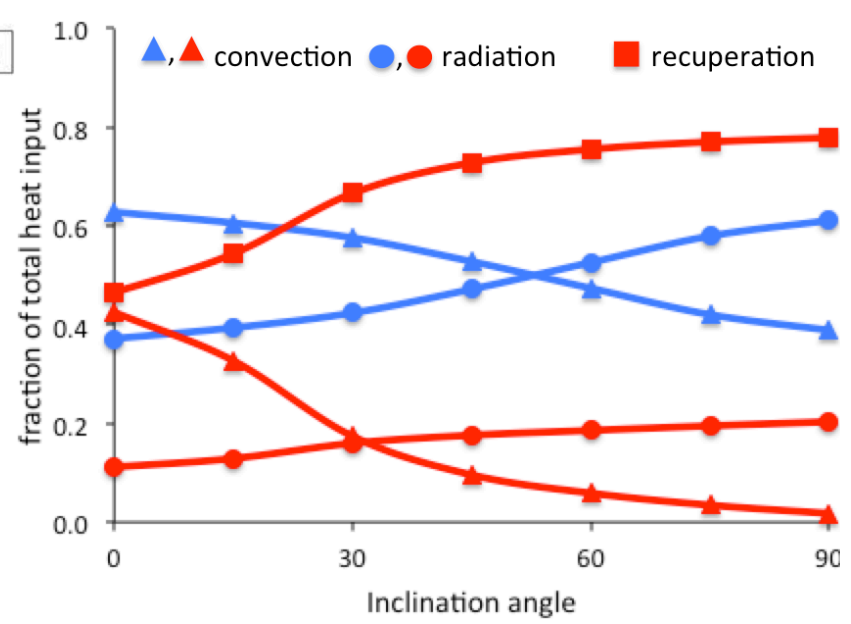

(b)

FIGURE 2. (a) Schematic diagram of scale-model cavity receiver apparatus used to investigate the effect of heat recuperation. Electrical heating is applied over the actively-heated section, and recuperation of heat occurs by conduction through the passive section. (b) Apportionment of cavity aperture thermal loss for cylindrical cavity without passive section (blue) and with passive section (red), i.e. the length to diameter ratio is one and two, respectively. Triangles and circles denote the proportion of the total heat input that is lost by the convective and radiative components, respectively, and squares denote the proportion of total heat input that is recuperated (when there is a passive section).

\section{ACTIVE AIRFLOW TO REDUCE CONVECTIVE LOSSES}

We examine here the possibility of managing the airflow in the vicinity of a cavity receiver to disrupt the exit of buoyant air from the cavity interior and thus reduce the losses of thermal energy. Attention in the initial phase of this study has focussed on use of a planar jet directed approximately across the cavity aperture (termed an "air curtain" or "aerowindow"). Air curtains are a familiar technology in a number of other semi-enclosed environments that include shop doorways, refrigerated display cases, clean rooms and industrial cool rooms or furnaces. However, knowledge of these established applications offers limited guidance for interaction of an air curtain with the convective flow in a cavity receiver, where the effects of geometry, inclination and high temperature differences play a role. 


\section{Laboratory Experiments}

A set of laboratory experiments was undertaken to demonstrate qualitatively that an air curtain could improve the thermal performance of a cavity receiver. A scale-model receiver consisting of an optically transparent rhomboidal cavity $200 \mathrm{~mm} \times 200 \mathrm{~mm} \times 100 \mathrm{~mm}$ was used to visualise the convective flow and to quantify the effect that an air curtain has in retaining buoyant fluid in the cavity (Fig. 3). The model receiver was immersed in a large saline water bath, and fresh water (marked by red dye) was introduced into the stagnation region of the cavity. The flow parameters (e.g. the salinity difference) were adjusted so that the convective flow mimicked approximately the conditions under which a cavity receiver operates in air, i.e. the Rayleigh number is of the same order - typically of $\mathrm{O}\left(10^{10}-10^{11}\right)$ - in both cases (cf. [15]). An "air curtain" of ambient water was directed across the aperture from a planar jet source of width $2 \mathrm{~mm}$ situated at both the upper (Fig. 3b) and lower (not shown) edges of the cavity. Both the direction and strength of the planar jet were varied. Optical measurements of the amount of dye retained in the cavity (e.g. [16]) were used to assess the relative benefit of the air curtain: an increased amount of buoyant fluid in the cavity is assumed to be a proxy for higher average temperature in the cavity and thus a reduction in convective heat loss from the interior cavity surfaces.

The experiments offer numerous useful physical insights, even though the water-immersed model receiver does not represent specifically the additional dynamical effect on the inertia balance of a large temperature difference in the flow. The results suggest there to be an optimum set of operating conditions for the aperture jet that maximises the amount of dye held in the cavity at a given cavity inclination angle. In general, an aperture jet situated at the upper edge of the cavity with a downward directed component of momentum performs best. Buoyant fluid that would be lost from the cavity is instead entrained into the turbulent aperture jet, carried downwards and recirculated through the cavity (Fig. 3b). The retention of buoyant fluid in the cavity increases with jet strength until a maximum performance improvement is reached. Beyond this point, the jet is observed to overly disturb the buoyant fluid throughout the cavity; indeed a sufficiently strong jet acts to increase convection losses by flushing buoyant fluid out of the cavity.

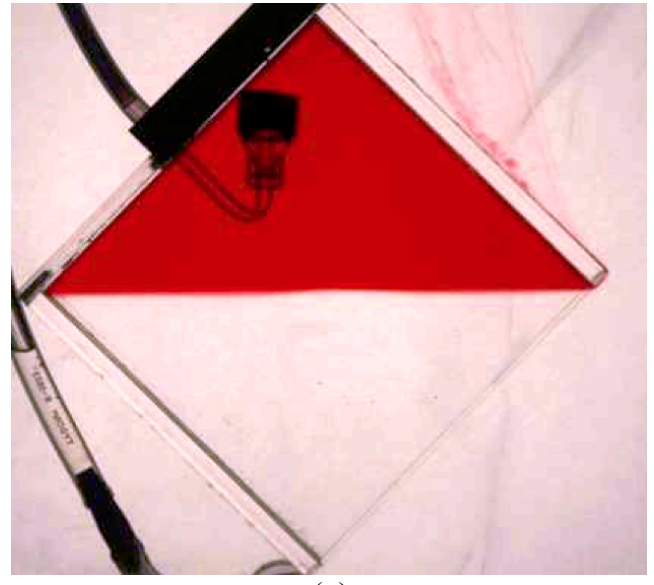

(a)

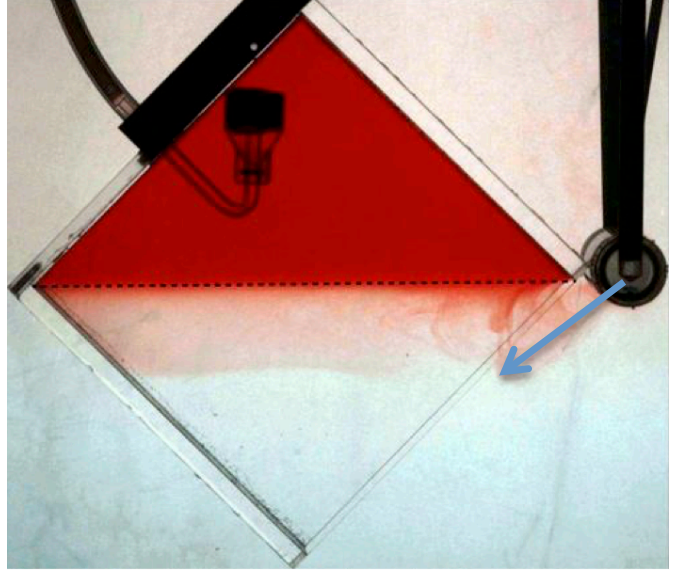

(b)

FIGURE 3. Qualitative demonstration of how an air curtain can modify flows in a cavity receiver. The optically transparent rhomboidal cavity model has an axis angled at $45^{\circ}$ from the horizontal (with aperture at the lower right) and is immersed in a large water bath. Red dye is used to mark buoyant fluid (i.e. the "hot air"), which is trapped by the shape of the cavity in a stagnation region and continually replenished by the source visible towards the top of the volume: (a) without an air curtain, buoyant fluid escapes from the receiver aperture and can be seen rising from the outer surface of the model at the upper right of the photo; (b) with a well-performing air curtain (directed approximately as shown by the arrow), considerably more buoyant fluid can be retained in the cavity.

\section{Computational Fluid Dynamics Modelling}

A set of three-dimensional high-resolution CFD simulations was conducted for the cylindrical model cavity studied by Taumoefolau et al. [8] (70 $\mathrm{mm}$ inner diameter and $150 \mathrm{~mm}$ in length) and similar to that used by AbbasiShavazi et al. [14]. The simulations reported here were carried out using a transient and compressible solver in 
ANSYS Fluent v. 15, and full details may be found in [17] (A closely-related companion study using twodimensional high resolution CFD simulations in OpenFOAM was also carried out, and details may be found in [18]). The receiver geometry was embedded in a significantly larger "ambient" domain with closed boundaries. The overall computational domain was discretised using a total of 340000 elements in a hybrid mesh incorporating structured and unstructured regions inside and outside the cavity, respectively. As above, the maximum resolution was placed inside the cavity; mesh-invariant solutions were found once the size of the elements near the walls was decreased to $4 \mathrm{~mm}$. Again, the highly-resolved computations did not require a turbulence closure scheme. In all simulations, the wall temperature inside the cavity was uniform and set to $720 \mathrm{~K}$, the ambient temperature was $300 \mathrm{~K}$ and no-slip conditions were imposed on the walls. Each simulation was continued until a confidence test indicated the solution had reached a statistically equilibrated state.

The air curtain studied here consisted of a planar jet of ambient air directed approximately across the aperture from a source whose width was fixed at $5 \mathrm{~mm}$. The jet speed and initial angle with respect to the aperture plane was varied parametrically for inclinations of the cavity axis up to $45^{\circ}$ from the horizontal. A summary of these results are given by Zhang et al. [17]; here we use the example of a cavity inclined at $45^{\circ}$ from the horizontal to highlight the important physics governing the interaction between the air curtain and the cavity interior in all cases examined.

The results confirm that air curtain operation can reduce convective losses from the cavity receiver, but that losses can also be increased when the aperture jet is too strong or misdirected. For a given cavity inclination, two distinct modes of optimal operation (termed "sealing" modes) are found that offer a similar reduction of convective losses. We term one the "partially-sealed" mode shown in Fig. 4b, which corresponds with the experimental visualisation in Fig. $3 \mathrm{~b}$. The air curtain is directed up to a few degrees either side of the aperture plane and acts like a virtual wall, allowing the stagnation region in the cavity to extend downward (cf. Fig. 4a, where there is no air curtain). However, entrainment of hot air counteracts the initial momentum of the jet before it can traverse the aperture, resulting in the weak plume that escapes upward at the bottom right of Fig. 4b. The second "fully sealed" mode (Fig. 4c) corresponds to a stronger jet that traverses the aperture somewhat unaffected by the entrainment of hot air. The air curtain is initially directed outward from the cavity, but is drawn back towards the aperture plane by relatively low pressure in the cavity to create the aerodynamic seal. The normal convective flow (Fig. 4a) is completely suppressed in both modes, and the heat loss is instead associated with entrainment into the turbulent planar air curtain jet. Although the convective loss is reduced by approximately $50 \%$ with either mode in the case shown here (and indeed for the broader range of cavity axis inclinations examined [17]), the fully-sealed mode is predicted to be more sensitive to the operating parameters of the air curtain. Preliminary estimates suggest that the parasitic energy consumed by operating an air curtain in either sealing mode will be at least two orders of magnitude smaller than the energy saved.

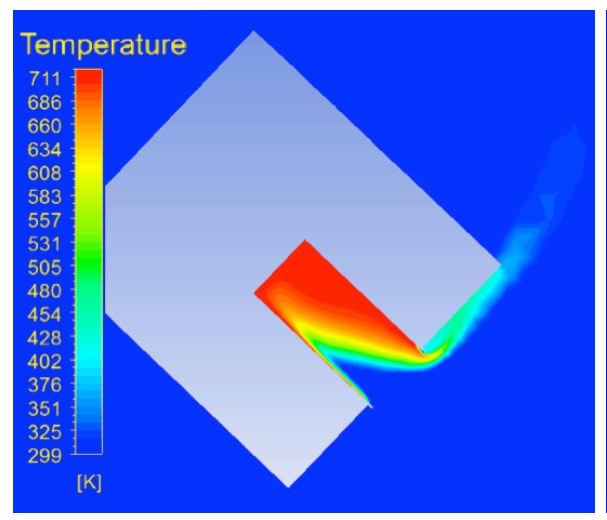

(a)

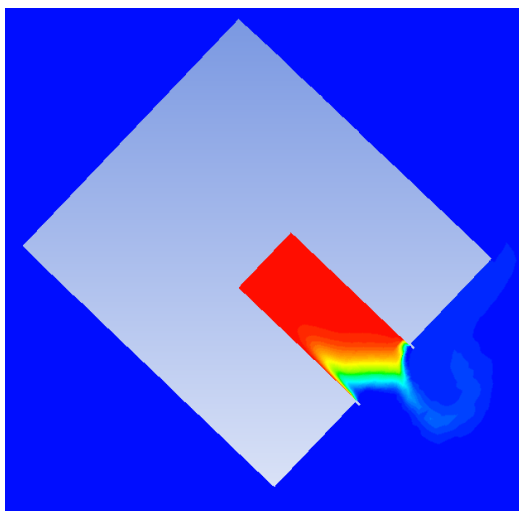

(b)

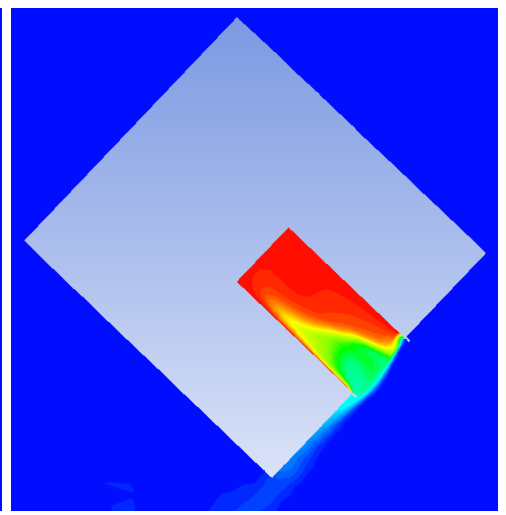

(c)

FIGURE 4. Temperature field (centre-plane) from CFD simulations of a cylindrical cavity receiver with axis inclined at $45^{\circ}$ to the horizontal and with a uniform imposed temperature of $720 \mathrm{~K}$ on the interior walls (see also [17]): (a) without an aperture air jet, hot air escapes from the cavity in a buoyant plume; (b) and (c) an air jet is directed across the aperture from the upper edge and two modes of "sealing" the cavity are observed (corresponding respectively to different jet speeds and angles of incidence with respect to the aperture plane). 


\section{CONCLUDING REMARKS}

We have examined two methods of reducing convective losses from a high temperature solar cavity receiver. The first method makes use of convection to redistribute a significant amount of heat within the cavity, thereby reducing the losses that would otherwise occur to the external environment. Receiver design plays an important role in this process because it is advantageous for the wall temperature to decrease towards the cavity aperture. Moreover, such a design can also work to lower the overall thermal losses, by deflating the wall temperature in the region where a greater proportion of the local emission can escape directly through the aperture. Thus, directing the coolest heat transfer fluid to the aperture section of the cavity wall helps maintain such a temperature distribution and reduce the convective, radiative and overall losses.

The second method employs an air curtain, consisting of a planar jet directed approximately across the cavity aperture, to disrupt the convective flow that would otherwise occur. Reductions in convective losses on the order of $50 \%$ are found to be achievable across a range of cavity inclinations. However, optimum reduction of convective losses requires control of both the aperture jet speed and direction. Ongoing work is examining the combination of these methods of loss reduction in conjunction with active extraction or injection of air into the receiver. The use of these techniques to partially shield the receiver from detrimental effects of ambient wind is also being investigated.

\section{ACKNOWLEDGEMENTS}

The research was supported by Australian Renewable Energy Agency USASEC Project grant 1-UFA006. GH was supported by ARC Future Fellowship FT10010086. This computational component of this research was undertaken on the NCI National Facility in Canberra, Australia, which is supported by the Australian Commonwealth Government.

\section{REFERENCES}

1. K. Lovegrove, G. Burgess and J. Pye, Sol. Energy 85, 620-626 (2011).

2. C. K. Ho and B. D. Iverson, Renewable and Sustainable Energy Reviews 29, 835-846 (2014).

3. J. Karni, A. Kribus, B. Ostraich and E. Kochavi, J. Sol. Energy Eng. 120, 101-107 (1998).

4. G. Maag, C. Falter and A. Steinfeld, J. Sol. Energy Eng. 133, 014501 (2010).

5. M. Röger, R. Buck and H. Müller-Steinhagen, J. Heat Transfer 127, 863-876 (2005).

6. J. I. Zapata, C.-A. Asselineau, J. D. Pye, M. Kaufer and G. O. Hughes, "An Integrated Optical and Thermal Model of Cavity Receivers for Paraboloidal Dish Concentrators", in Proc. 2014 Asia-Pacific Solar Research Conference, Sydney, December (2014).

7. J. Pye, G. Hughes, E. Abbasi, C.-A. Asselineau, G. Burgess, J. Coventry, M. Kaufer, F. Venn and J. Zapata, "Development of a higher-efficiency tubular cavity receiver for direct steam generation on a dish concentrator", in Proc. SolarPACES 2015, Cape Town, October (2015).

8. T. Taumoefolau, S. Paitoonsurikarn, G. Hughes and K. Lovegrove, J. Sol. Energy Eng. 126, 801-807 (2004).

9. S. Paitoonsurikarn, K. Lovegrove, G. Hughes and J. Pye, J. Sol. Energy Eng. 133, 021004 (2011).

10. R. D. Jilte, S. B. Kedare and J. K. Nayak, Mech. Eng. Res. 3, 25-43 (2013).

11. S. Y. Wu, J. Y. Guan, L. Xiao, Z. G. Shen and L. H. Xu, Experimental Thermal and Fluid Science 45, 92-101 (2013).

12. K. Lovegrove, T. Taumoefolau, S. Paitoonsurikarn, P. Siangsukone, G. Burgess, A. Luzzi, G. Johnston, O. Becker, W. Joe and G. Major, "Paraboloidal Dish Solar Concentrators for Multi-Megawatt Power Generation", in Proc. ISES Solar World Congress, Sweden, June, (2003).

13. A. M. Clausing, Sol. Energy 27, 295-300 (1981).

14. E. Abbasi-Shavazi, G. O. Hughes and J. D. Pye, Energy Procedia 69, 269-278 (2015).

15. P. F. Linden, Annu. Rev. Fluid Mech. 31, 201-238 (1999).

16. J. M. Holford and S. B. Dalziel, Appl. Sci. Res. 56, 191-2-7 (1996).

17. J. J. Zhang, J. D. Pye and G. O. Hughes, "Active air flow control to reduce cavity receiver heat loss", in Proc. Ninth International Conference on Energy Sustainability, ASME Power \& Energy, San Diego, June (2015).

18. A. McIntosh, G. O. Hughes and J. Pye, "Use of an Air Curtain to Reduce Heat Loss from an Inclined OpenEnded Cavity", in Proc. Ninteenth Australasian Fluid Mechanics Conference, Melbourne, December (2014). 